\title{
Bee pollen properties: uses and potential pharmacological applications-a review
}

\begin{abstract}
Bee pollen is a food supplement widely used in the world due to the benefits promoted by the bioactive compounds present in it. In addition to the very attractive nutritional composition, the presence of phenolics, flavonoids, terpenes are responsible for the antioxidant activity, antimicrobial, antiinflammatory, possible action on benign prostatic hyperplasia, no cytotoxic. It is intended to show with this article the uses and potential of bee pollen application. Numerous studies are aimed at identifying these compounds in pollen samples around the world, because according to the region, climatic type, seasons, shift and bee species, the composition is influenced by the variety. The use of pollen is more recurrent in the food industry are comprehensively summarized in this review, but the biomedical could also take advantage of the potential that this product demonstrates.
\end{abstract}

Keywords: bee products, bioactive compounds, antioxidant activity, pharmacological uses
Volume 7 Issue 5 - 2018

\author{
Afra M C B Nascimento,' Geraldo E Luz Jr² \\ 'RENORBIO, Federal University of Piaui, Brazil \\ ${ }^{2}$ Department of Chemistry, State University of Piaui, Brazil
}

Correspondence: Geraldo E Luz, Department of Chemistry, State University of Piaui, Rua João Cabral, S/N, Pirajá,Teresina-PI, Brazil Tel +5586 32137942, Email geraldoeduardo@gmail.com

Received: June 06, 2018| Published: September 04, 2018

\section{Introduction}

Foods are natural source of several health promoting substances with significant biological activities. ${ }^{1}$ Recently, dietary flavonoids are attracting increasing interest based on in vitro and in vivo studies suggesting a variety of beneficial biological properties. ${ }^{2}$ The bee pollen is one of bee products that has highlighted due to the presence of bioactive compounds associated with health benefits. ${ }^{3}$

Bee pollen is a mixture of flower pollen, nectar and bee secretions. It can be collected by beekeepers without damage to the beehive. This natural product, that has been gaining prominence, is recognized to be a valuable apitherapeutic product with potential for medical and nutritional applications. ${ }^{4}$ In fact it contains proteins, the 22 basic amino acids, carbohydrates, lipids, vitamins and minerals. ${ }^{5}$

The significative amount of phytosterols, carbohydrates, enzymes, nucleic and triterpene acids, vitamins and other biologically active substances in bee pollen provides anti-inflammatory, wound healing, cardiotonic, antiatherosclerotic and other pharmacological actions. ${ }^{6}$ Thereby bee pollen is considered as "only perfectly complete food".

The most important bioactive substances in bee-pollen are phenolic compounds and carotenoids. Phenolic compounds are responsible for the color of the grain and its characteristic bitter taste. ${ }^{8}$ Carotenoids are also important for color and for other biological functions, such as antioxidant activity, provitamin A activity, and enhancement of the immune system.

Nowdays, the major use of bee pollen is specialty food supplement but it has potential therapeutic benefits. Particularly, it has been used for a long time to manage benign prostatic hyperplasia (BPH) which is the most common disorder for older men, ${ }^{9}$ how demonstrated by Park et al., ${ }^{10}$ that obtained satisfactory results using a herbal composition with bee pollen and Panax ginseng (ginseng) to the treatment of BPH. Therefore it is intended to show with this article the uses and potential of bee pollen application.

\section{Bioactive compound, antioxidant activity and others effects}

The chemical composition of bee pollen is directly influenced by the botanical diversity from which it was collected, as shown by Modro et al. ${ }^{11}$ In addition to the botanical contribution, storage time, nutritional status of the plant and environmental conditions in the phase of pollen collection by bees also influence the composition. ${ }^{3}$ It should be noted that the action of a natural antioxidant depends on several factors, such as: reaction mechanism, experimental conditions and matrix heterogeneity. ${ }^{5}$

LeBlanc et al., ${ }^{12}$ identified $p$-hydroxybenzoic, $p$-coumaric, vanillic, gallic and ferulic acids in bee pollen from the United States. Menezes maintains that among the carotenoids found in bee pollen, $\alpha$-carotene and $\beta$-carotene are predominant, and in a smaller amount cryptoxanthin, xanthophylls and flavoxanthin are predominant. It should be emphasized that the flavonoid and phenolic profile, both in quantity and variety, is directly related to the pollen types that make up the bee pollen.

Fanali, Dugo and $\operatorname{Rocco}^{1}$ found nine polyphenols: $o-$, $p$-coumaricacid, ferulic acid, myricetin, cinnamic acid, quercetin, naringenin, hesperitin and kaempferol. All analytes, with the exception of $p$-coumaric acid and myricetin, which partially co-eluted with other pollen components, were also quantified in the sample. Lv et al. ${ }^{14}$ identified pollen samples from the Qinghai-Tibetan Plateau by HPLC-DAD-APCI/MS, quercetin and campperol, with no routine and isoramnetin being detected.

Six 4-desmethylsterols, one 4-monomethylsterol, four 4, 4-dimethylsterols and another three triterpene alcohols were reported for the first time in the bee pollen. Significant difference was found in the contents of free and esterified triterpene alcohols between beecollected pollens from the selected plant origins. The total triterpene alcohol levels in bee pollen oil fractions have been found to vary between $2.6 \mathrm{~g} / 100 \mathrm{~g}$ and $20.4 \mathrm{~g} / 100 \mathrm{~g}$ oil. Triterpene alcohols in the bee pollen oils were dominated by steryl esters $(>67 \%)$. Concurrently, 
esterified triterpene alcohols in lotus bee pollen oil fraction predominantly consisted of large amount of cycloartenol. ${ }^{15}$

Carpes et al., ${ }^{16}$ evaluated the antioxidant activity after the use of Amberlite XAD2 resin for the purification of samples of bee pollen extracts, and even occurring a decrease of half of the phenolic compounds, the antioxidant activity was effective, by the DPPH method. In the case of $\beta$-carotene, a possible explanation is that the slight increase in antioxidant activity may have been the removal of polar compounds present in the extract, which in turn are more diluted, having a lower efficiency in the protection against lipid oxidation.

Fatrcová- ${ }^{-S r a m k o v a ́ ~ e t ~ a l ., ~}{ }^{17}$ tested the antioxidant profile only in ethanolic extract of monofloral pollen of poppy, canola and sunflower, verifying the best values for all parameters in canola pollen samples. The results of antioxidants obtained by Mohdaly et al., ${ }^{18}$ showed that the radical ABTS test showed better activity than DPPH, such as the radical stereoselectivity and the solubility of the extract in different test systems as influencers of the extract's ability to react and eliminate different radicals.

The anti-inflammatory activity was assessment by indirect method of measuring the effect of inhibition of the reaction of catalysis by hyaluronidase, the results obtained were compared with those of another study with propolis, due to lack of studies with the same material, however the values for this aspect were smaller than those of propolis, where the researchers associated this fact with the lowest phenolic content. ${ }^{19}$

In the study about bioactivity of Eucalyptus globulus and Salix atrocinerea bee pollen extracts on rats showed that both species have antidiarrheal properties, reducing diarrheic excrement by about $30 \%$. It be concluded that activity may be due to the polyphenolic constituents, especially quercetin and its derivatives, although other compounds can play a role in this activity and also be responsible for differences in results. ${ }^{20}$

\section{Pharmacological use}

A broad range of therapeutic properties have been suggested to bee pollen, such as: antimicrobic, antioxidant, hepatoprotective, chemopreventive and anticarcinogenic, antiatherosclerotic, antiinflammatory, antiallergenic, and immunomodulatory activities..$^{21,22}$

According to the authors ${ }^{6}$ a composition of standardized substances of bee products - PH, PPHD and BP, are a promising source for development of new domestic drugs for use in urological practice.

Bharti et al., ${ }^{23}$ supplemented the diet of rats with bee pollen which showed antioxidant and protective effect when given along with antituberculosis drugs (rifampicin as well as isoniazid). It is thus observed that bee pollen has strong potential to provide protection against antituberculosis drugs-induced toxicity in rats as evidenced by improved histological parameters and rebuilding of the antioxidant/ oxidative status.

Omar et al., ${ }^{24}$ from your research, suggested that BPE of Malaysian stingless bee, L. terminata is a potential chemopreventive agent and can be used as a supplementary treatment for chemotherapy drugs. BPE might be able to be used to potentiate the effect of chemotherapy drugs with the possibility to reduce the required dose of the drugs. Huang et al., ${ }^{25}$ reinforce this information, indicating the use of compound SCBPE could reduce the damage of liver and kidney caused by cisplatin by reducing the level of oxidative stress, inflammation and apoptosis, and improving the antioxidant, anti-inflammatory and anti-apoptotic capacity of the body.

Furthermore, bee pollen as supplement can be combined with chemotherapy treatment to treat side effects of cancer as reported in a recent study by Salles et al. ${ }^{26}$ which showed that bee pollen was able to improve muscle mass and metabolism in undernourished rats.

Yildiz et al. ${ }^{27}$ showed that use of bee pollen accelerated recovery from liver damage caused by carbon tetrachloride poisoning and it was therefore proposed as an alternative therapy for liver damage. Kolesarova et al., ${ }^{28}$ studied the effects of bee pollen on ovarian activity and showed that bee pollen is a strong regulator of ovarian activity in rats. Karampour, Hemmati, MAamir (2016) can be concluded that hydroalcoholic extract of bee pollen has anxiolytic properties through bonding to benzodiazepines in gamma-aminobutyric acid receptor, and thus moderates anxious reactions.

Mohamed et al., ${ }^{29}$ assessed the effects of bee pollen and/or date palm pollen suspensions on the glycemic state, testicular dysfunctions, oxidative stress and antioxidant defense system in diabetic male Wistar. It is may be attributed to the improvements in the glycemic state secondary to ameliorations in the insulin secretion and beta cell function as well as to enhancement of the testicular antioxidant defense system.

Al-Salem et al., ${ }^{30}$ and El-Ansary et al., ${ }^{31}$ suggest that bee pollen has potential candidate for further studies for the development of new treatment strategies to autism. In the both studies was proved the bee pollen positive effect due to the biological properties of its components, in particular, phenolic compounds and amino acid composition, resulting to ameliorate oxidative stress, neuroinflammation, poor detoxification, and abnormal gut microbiota.

Cornara et al., ${ }^{32}$ emphasize that studies aimed at refining the knowledge of action mechanisms from bee products are pivotal importance for developing applications of honeybee products to medicinal uses, because the complexity and variability in composition of these products raise the need of their standardization before safe and predictable clinical uses can be achieved.

The extracts of bee pollen collected from flowers of different angiosperms can be regarded as a promising therapeutic and nutritional natural food supplement. Its functional biological property is due to the high content of flavonoids and polyphenols and considerable radical scavenging capacity. However, more research and more experimental and clinical studies are required to verify the effectiveness of bee pollen extracts, using tests accepted by the Western medical establishment. ${ }^{22}$

\section{Conclusion}

The bee pollen is a rich product in bioactive compounds which are associated innumerable therapeutic effects that be used to development food products or pharmaceutical. Due to many variations in function soil and climatic conditions, botanical source and storage and processing conditions, is relevant their phytochemical and botanical characterization.

Although there are no available product based-bee pollen commercially for pharmacological purposes it is noted that the scientific field has worked hard to elucidate the effects and action mechanisms the bee products. Thus new research on the characterization and application of the therapeutic properties of bee 
pollen is strongly encouraged.

\section{Acknowledgements}

The authors thank the CAPES and CNPq the financial support.

\section{Conflict of interests}

Author declares that there is no conflict of interest.

\section{References}

1. Fanali C, Dugo L, Rocco A. Nano-liquid chromatography in nutraceutical analysis:Determination of polyphenols in bee pollen. $J$ of Chromatography A. 2013;1313:270-274.

2. Kacaniova M, Rovna $\mathrm{K}$, Arpašova $\mathrm{H}$, et al. The effects of bee pollen extracts on the broiler chicken's gastrointestinal microflora. Research in Veterinary Science. 2013;95(1):34-37.

3. Sattler JAG, Melo ILP, Granato D, et al. Impact of origin on bioactive compounds and nutritional composition of bee pollen from southern Brazil: A screening study. Food Research International. 2015;77:82-91.

4. Almeida-Muradian LB, Pamplona LC, Coimbra S, et al. Chemical composition and botanical evaluation of dried bee pollen pellets. Journal of Food Composition and Analysis. 2005;18(1):105-111.

5. Morais M, Moreira L, Feas X, et al. Honeybee-collected pollen from five Portuguese Natural Parks: Palynological origin, phenolic content, antioxidant properties and antimicrobial activity. Food and Chemical Toxicology. 2011;49:1096-1101.

6. Koval VM, Tykhonov OI, Shpychak OS. Study of specific pharmacological activity of standardized composition of bee product substances for treatment of urogenital system. Zaporozhye Medical Journal. 2017;19(5):6642-6646.

7. Kosti AZ, Bara MB, Stanojevi SP, et al. Physicochemical composition and techno-functional properties of bee pollen collected in Serbia. LWT - Food Science and Technology. 2015;62(1):301-309.

8. Bogdanov S. Contaminants of bee products. Apidologie. 2006;37:1-18.

9. Murakami M, Tsukada O, Okihara K, et al. Beneficial effect of honeybee-collected pollen lump extract on benign prostatic hyperplasia (BPH) - a double-blind, placebo-controlled clinical trial. Food Science and Technology Research. 2008;14:306-310.

10. Park HK, Kim SK, Lee SW, et al. A herbal formula, comprising Panax ginseng and bee-pollen, inhibits development of testosteroneinduced benign prostatic hyperplasia in male Wistar rats. Saudi Journal of Biological Sciences. 2017;24:1555-1561.

11. Modro AFH, Message D, Luz CFP, et al. Composição e qualidade de pólen apícola coletado em Minas Gerais. Pesq agropec bras Brasília. 2007;42(8):1057-1065.

12. Leblanc BW, Davis OK, Boue S, et al. Antioxidant activity of Sonoran Desert bee pollen. Food Chemistry. 2009;115:1299-1305.

13. Menezes JDS. Compostos bioativos do Pólen apícola. Dissertion. Federal University of Bahia: Salvador; 2009.

14. Lv H, Wang X, He Y. Identification and quantification of flavonoid aglycones in rape bee 4 pollen from Qinghai-Tibetan Plateau by HPLCDAD-APCI/MS. Journal of Food Composition and Analysis. 2014.

15. $\mathrm{Xu} X, \mathrm{Gao} \mathrm{Y}$, Sun L. Free and esterified triterpene alcohol composition of bee pollen from different botanical origins. Food Research International. 2012;48:650-656.
16. Carpes ST, Alencar SM, Cabral ISR. Polyphenols and palynological origin of bee pollen of Apis mellifera L. from Brazil. Characterization of polyphenols of bee pollen CyTA. Journal of Food. 2013;11(2):150-161.

17. Fatrcová-Sramková K, Nôzková J, Kacaniová M, et al. Antioxidant and antimicrobial properties of monofloral bee pollen. Journal of Environmental Science and Health. 2013;48:133-138.

18. Mohdaly AAA, Mahmoud AA, Roby MHH, et al. Phenolic extract from propolis and bee pollen: composition, antioxidant and antibacterial activities. Journal of Food Biochemistry. 2015;39(5):1745-4514.

19. Pascoal A, Rodrigues S, Teixeira A, et al. Biological activities of commercial bee pollens: antimicrobial, antimutagenic, antioxidant and anti-inflammatory. Food and Chemical Toxicology. 2014;63:233-239.

20. Frigerio C. Optimização e influência na bioactividade do processo de secagem por radiação infravermelha de amostras de pólen apícola. Dissertation. University of Porto: Lisboa; 2009.

21. Komosinska-Vassev K, Olczyk P, Kaźmierczak J, et al. Bee pollen chemical composition and therapeutic application. Evid based Complement Alternat Med. 2015:1-6.

22. Denisow B, Denisow-Pietrzyk M. Biological and therapeutic properties of bee pollen: a review. J Sci Food Agric. 2016;96(13):4303-4309.

23. Bharti U, Kumar NR, Kaur J. Modulatory activity of bee pollen against the toxicity of antituberculosis drugs rifampicin and isoniazid in testis of sprague dawley rats. Asian J Pharm Clin Res. 2017;10(9):161-163.

24. Omar WAW, Azhar NA, Fadzilah NH, et al. Bee pollen extract of Malaysian stingless bee enhances the effect of cisplatin on breast cancer cell lines. Asian Pac J Trop Biomed. 2011 6(3):265-269.

25. Huang H, Shen Z, Geng Q, et al. Protective effect of Schisandra chinensis bee pollen extract on liver and kidney injury induced by cisplatin in rats. Biomedicine \& Pharmacotherapy. 2017;95:1765-1776.

26. Salles J, Cardinault N, Patrac V, et al. Bee pollen improves muscle protein and energy metabolism in malnourished old rats through interfering with the Mtor signaling pathway and mitochondrial activity. Nutrients. 2014;6(12):5500-5516.

27. Yildiz O, Can Z, Saral O, et al. Hepatoprotective potential of chestnut bee pollen on carbon tetrachloride-induced hepatic damages in rats. Evid Based Complement Alternat Med. 2013:461478.

28. Kolesarova A, Bakova Z, Capcarova M, et al. Consumption of bee pollen affects rat ovarian functions. J Anim Physiol Anim Nutr (Berl). 2013;97(6):1059-1065.

29. Mohamed NA, Ahmed OM, Hozayen WG, et al. Ameliorative effects of bee pollen and date palm pollen on the glycemic state and male sexual dysfunctions in streptozotocin-Induced diabetic wistar rats. Biomed Pharmacother. 2018;97:9-18.

30. Al-Salem HS, Bhat RS, Al-Ayadhi L, et al. Therapeutic potency of bee pollen against biochemical autistic features induced through acute and sub-acute neurotoxicity of orally administered propionic acid. BMC Complement Altern Med. 2016;16(120):1-10.

31. El-Ansary A, A1-Salem HS, Asma A, et al. Glutamate excitotoxicity induced by orally administered propionic acid, a short chain fatty acid can be ameliorated by bee pollen. Lipids in Health and Disease. 2017;16(96):1-9.

32. Cornara L, Biagi M, Xiao J, et al. Therapeutic properties of bioactive compounds from different honeybee products. Front Pharmacol. 2017;8:412. 